\title{
Corrigendum to "Study of some equivalence classes of primes" [Notes on Number Theory and Discrete Mathematics, Vol. 23, 2017, No. 2, 21-29]
}

\author{
Sadani Idir \\ Department of Mathematics, \\ University of Mouloud Mammeri \\ 15000 Tizi-Ouzou, Algeria \\ e-mail: sadani.idir@yahoo.fr
}

It was discovered that the original online version of the paper "Study of some equivalence classes of primes" contained the following minor misprints:

- Page 27, Line 14. "less than or equal to $p_{0, x}$ " becomes "less than or equal to $\pi\left(p_{0, x}\right)$ ".

- Page 28, Line 6. " $\pi_{i t e r}(x)$ " becomes " $\pi_{c}(x)$ ".

As of March 2019, the correction has been made in the online version of the paper, available at:

http://nntdm. net/volume-23-2017/number-2/21-29/.

Unfortunately, the discovery of these misprints was too late to make correction in the printed issue. 